PHYSICAL REVIEW D 79, 035009 (2009)

\title{
Deciphering the spin of new resonances in Higgsless models
}

\author{
Alexandre Alves* and O. J. P. Éboli ${ }^{\dagger}$ \\ Instituto de Física, Universidade de São Paulo, São Paulo-SP, Brazil.
}

M. C. Gonzalez-Garcia

Institució Catalana de Recerca i Estudis Avançats (ICREA), Departament d'Estructura i Constituents de la Matèria, Universitat de Barcelona, 647 Diagonal, E-08028 Barcelona, Spain and C. N. Yang Institute for Theoretical Physics, SUNY at Stony Brook, Stony Brook, New York 11794-3840, USA

\section{J. K. Mizukoshi ${ }^{\S}$}

Centro de Ciências Naturais e Humanas, Universidade Federal do ABC, Santo André-SP, Brazil. (Received 19 October 2008; revised manuscript received 20 January 2009; published 13 February 2009)

\begin{abstract}
We study the potential of the CERN large hadron collider to probe the spin of new massive vector boson resonances predicted by Higgsless models. We consider its production via weak boson fusion which relies only on the coupling between the new resonances and the weak gauge bosons. We show that the large hadron collider will be able to unravel the spin of the particles associated with the partial restoration of unitarity in vector boson scattering for integrated luminosities of $150-560 \mathrm{fb}^{-1}$, depending on the new state mass and on the method used in the analyses.
\end{abstract}

DOI: 10.1103/PhysRevD.79.035009

PACS numbers: $12.60 . \mathrm{Fr}, 14.70 . \mathrm{PW}$

\section{INTRODUCTION}

Despite the success of the standard model (SM) of particle physics in describing electroweak physics below $\sim 100 \mathrm{GeV}$ in terms of a non-Abelian gauge theory with spontaneously broken $S U(2)_{L} \times U(1)_{Y}$ gauge group, the gauge symmetry does not predict the precise mechanism of the electroweak symmetry breaking (EWSB). Indeed, up to this moment, there is no direct experimental signal of the mechanism of EWSB, being its search one of the main goals of the large hadron collider (LHC).

The EWSB mechanism plays an important role in the high energy electroweak gauge boson scattering which violates partial wave unitarity or becomes strongly interacting at energies of the order of $E \sim 2 \mathrm{TeV}$, if there is no new state to cut off its growth [1,2]. In the context of the $\mathrm{SM}$, as well as in its supersymmetric realization, electroweak symmetry is broken by the vacuum expectation value of some weakly coupled neutral scalar state(s), the Higgs boson(s), which will contribute to electroweak gauge boson scattering, preventing the unitarity violation of the process.

Alternatively, Higgsless extensions of the SM [3-5] have been proposed in which the electroweak symmetry is broken without involving a fundamental Higgs field. Generically on these models, the electroweak symmetry is broken by boundary conditions in a higher dimensional space. The originally proposed Higgsless models gave large contributions to precision electroweak observables,

\footnotetext{
*aalves@fma.if.usp.br

†eboli@fma.if.usp.br

*concha@insti.physics.sunysb.edu

§mizuka@ufabc.edu.br
}

in particular, to the $S$ parameter [6] $\left(\epsilon_{3}\right.$ [7]) [8-13]. Such problems could be overcome, for example, by appropriate modifications of the fermion sector. In this way, a variety of Higgsless models have been constructed [14-24] which ensure agreement with electroweak precision data.

From the point of view of unitarity, all Higgsless models share the common feature that new weakly interacting spin-1 gauge bosons particles with the same quantum numbers as the SM gauge bosons appear and they are responsible for the partial restoration of unitarity in vector boson scattering and for rendering a theory weakly coupled to energies well above $2 \mathrm{TeV}$ [25-27]. This property allows for an almost model independent search for the lightest charged resonance $V_{1}^{ \pm}$at the LHC through $p p \rightarrow V_{1}^{ \pm} W^{\mp}$ or via weak boson fusion $p p \rightarrow V_{1}^{ \pm} q q[28,29]$, as long as $V_{1}^{ \pm}$remains a narrow resonance. The LHC experiments will be able to unravel the existence of the charged state via these processes with modest integrated luminosities of $10-60 \mathrm{fb}^{-1}$. On the contrary, the corresponding search for the neutral vector resonance in gauge boson fusion is expected to be very difficult, since a generic feature of this class of models is the absence of coupling between the neutral resonance and $Z Z$ pairs. Reconstructing the heavy neutral vector resonance decaying into $W^{+} W^{-}$requires at least one hadronic $W$ decay, posing the challenge to dig it out from the large SM backgrounds.

Once a clear signal of the charged resonance is observed in the above channels, it is mandatory to study its spin to confirm that the new state is indeed a vector particle. In this work, our goal is to probe the $V_{1}^{ \pm}$spin via the study of weak boson fusion production of $V_{1}^{ \pm}$with its subsequent decay into leptons, i.e. 


$$
p p \rightarrow V_{1}^{ \pm} j j \rightarrow \ell^{ \pm} \nu \ell^{\prime+} \ell^{\prime-} j j
$$

with $\ell$ and $\ell^{\prime}=e$ or $\mu$, considering final states where the $W$ 's and $Z$ 's decay into different and same flavor charged leptons. To determine the spin of the state decaying into $W^{ \pm} Z$ we contrast the final state distributions arising from the production and decay of the vector charged state with the ones stemming from the decay of a scalar state; i.e. we work in the framework commonly used to analyze the spin of supersymmetric particles [30,31]. Here we show that it is possible to determine the spin of a new heavy resonance decaying into $W^{ \pm} Z$ at the LHC with $99 \%$ CL for luminosities of $\sim 150-560 \mathrm{fb}^{-1}$, depending on the particle mass and the method used in the analysis.

\section{MODEL AND CALCULATION SETUP}

The restoration of partial wave unitarity in Higgsless models is due to new Kaluza-Klein resonances $V_{(i)}^{ \pm}$and $V_{(i)}^{0}$. The couplings $V_{(i)}^{+} W^{-} Z\left(V_{(i)}^{0} W^{-} W^{+}\right)$have the same Lorentz structure of the SM $W^{+} W^{-} Z$ vertex with a coupling constant $g_{V W Z}^{(i)}\left(g_{V W W}^{(i)}\right)$. In order to cancel the dangerous terms in the scattering $W Z \rightarrow W Z$ that depend on $E^{2}$ and $E^{4}$, where $E$ is the energy of the incoming $W$ and $Z$ in the center-of-mass system, the new vector state coupling constants must satisfy the following constraints:

$$
\begin{gathered}
g_{\mathrm{WWZZ}}=g_{\mathrm{ZWW}}^{2}+\sum_{i}\left(g_{\mathrm{VWZ}}^{(i)}\right)^{2}, \\
2\left(g_{\mathrm{WWZZ}}-g_{\mathrm{ZWW}}^{2}\right)\left(M_{W}^{2}+M_{Z}^{2}\right)+g_{\mathrm{ZWW}}^{2} \frac{M_{Z}^{4}}{M_{W}^{2}} \\
=\sum_{i}\left(g_{\mathrm{VWZ}}^{(i)}\right)^{2}\left[3\left(M_{i}^{ \pm}\right)^{2}-\frac{\left(M_{Z}^{2}-M_{W}^{2}\right)^{2}}{\left(M_{i}^{ \pm}\right)^{2}}\right] .
\end{gathered}
$$

Equations (2) and (3) constrain the couplings of the lightest charged Kaluza-Klein state to $W Z$ pairs,

$$
g_{\mathrm{VWZ}}^{(1)} \lesssim \frac{g_{\mathrm{ZWW}} M_{Z}^{2}}{\sqrt{3} M_{1}^{ \pm} M_{W}} .
$$

In our analysis we assume that this bound is saturated [32], which leads to the largest allowed value for $g_{\mathrm{VwZ}}^{(1)}$, and we evaluate the quartic coupling $g_{\text {wwzZ }}$ using Eq. (2). Moreover, we assume that the $V_{1}^{ \pm}$couplings to fermions are small and that the $V_{1}^{ \pm}$'s mainly decay into $W Z$ pairs. This hypothesis is, in fact, realized in some Higgsless models [24].

Our study of the $V_{1}^{ \pm}$spin was carried out by comparing the kinematic distributions of its decay products with the predictions for the production of a spin-0 resonance. Since the signal for the new charged state is characterized by peak in the $W Z$ invariant mass distribution, we use as template the kinematic distributions in a model which is the SM without a Higgs plus a scalar charged state, $H^{ \pm}$, with an interaction $H^{ \pm} Z^{\mu} W_{\mu}^{\mp}$. The coupling of the
$H^{ \pm} Z^{\mu} W_{\mu}^{\mp}$ vertex is chosen such that the $H^{ \pm}$production cross section is equal to the one for $V_{1}^{ \pm}$after all cuts. We also set the $H^{ \pm}$width equal to the $V_{1}^{ \pm}$one.

We performed a parton level study using the full tree level amplitude for the final state processes in order to keep track of spin correlations. The matrix elements were generated using the package MADGRAPH [33], where we included the Higgsless (and template) model particles and interactions. We employed the CTEQ6L parton distribution functions [34] with the factorization scale $\mu_{F}=$ $\sqrt{\left(p_{T j 1}^{2}+p_{T j 2}^{2}\right) / 2}$, where $p_{T j i}$ are the transverse momenta of the tagging jets. For the QCD backgrounds we chose the renormalization scale $\mu_{R}=\mu_{F}$. In order to have a crude simulation of the detector performance we smeared energies, but not directions, of all final state partons with a Gaussian error. For the jets, we assumed a resolution $\Delta E / E=0.5 / \sqrt{E} \oplus 0.03$, if $\left|\eta_{j}\right| \leq 3$, and $\Delta E / E=$ $1 / \sqrt{E} \oplus 0.07$, if $\left|\eta_{j}\right|>3$ ( $E$ in $\mathrm{GeV}$ ), while for charged leptons we used a resolution $\Delta E / E=0.1 / \sqrt{E} \oplus 0.01$. Furthermore, we considered the jet tagging efficiency to be $0.75 \times 0.75=0.56$, while the lepton detection efficiency is taken to be $0.9^{3}=0.73$.

\section{RESULTS}

We analyzed the process

$$
p p \rightarrow \ell^{ \pm} \ell^{\prime+} \ell^{\prime-} j j+\not E_{T} \text { with } \ell, \ell^{\prime}=e, \mu,
$$

which contains the contribution of the vector boson fusion production of new charged resonances decaying into leptons; see Eq. (1). This process possesses a significant irreducible background originating from electroweak and QCD $W Z j j$ production. Moreover, the production of $t \bar{t}$ pair in association with a jet exhibits a large cross section after we demand the presence of two tagging jets [35] and can lead to trilepton events when both $t$ 's decay semileptonically and the decay of one of the $b$ 's leads to an isolated lepton. ${ }^{1}$

Initially we imposed the following jet acceptance cuts designed to enhance events produced by vector boson fusion,

$$
\begin{aligned}
p_{T}^{j} & >20 \mathrm{GeV}, \quad\left|\eta_{j}\right|<4.9, \\
\left|\eta_{j 1}-\eta_{j 2}\right| & >3.8, \quad \eta_{j 1} \cdot \eta_{j 2}<0 .
\end{aligned}
$$

We also applied lepton acceptance and isolation cuts

$$
\begin{aligned}
\left|\eta_{\ell}\right| \leq 2.5, & p_{T}^{\ell} \geq 10 \mathrm{GeV} \\
\Delta R_{\ell j} \geq 0.4, & \Delta R_{\ell \ell} \geq 0.4 .
\end{aligned}
$$

As we can see from Table I the SM background is still quite large after these cuts with the $t \bar{t} j$ production being the

\footnotetext{
${ }^{1}$ We considered a lepton to be isolated if the hadronic energy deposited in a cone of size $\Delta R<0.4$ is smaller than $10 \mathrm{GeV}$
} 
dominant contribution. In order to reduce this background we explore two features of the signal and backgrounds. First of all, in the $t \bar{t} j$ production the lepton coming from the $b$ semileptonic decay is quite soft, therefore, it can be reduced by imposing an additional lepton transverse momentum cut:

$$
p_{T}^{\ell}>25 \mathrm{GeV} .
$$

Moreover, two of the leptons in the signal come from a $Z$ decay, consequently we also required that the events present a pair of same flavor opposite charge leptons (SFOC) with an invariant mass in a window around the $Z$ mass. Thus we further demanded

$$
\left|M_{\ell \ell}^{\mathrm{SFOC}}-M_{Z}\right|<10 \mathrm{GeV} \text {. }
$$

The presence of just one neutrino in the signal final state, Eq. (1), allows for full reconstruction of the neutrino momentum - up to a twofold ambiguity on its longitudinal component-by imposing the transverse momentum conservation and requiring that the invariant mass of the neutrino- $\ell^{ \pm}$pair, where $\ell$ is the charged lepton not identified as coming from the $Z$ decay, is compatible with the $W$ mass:

$$
\begin{aligned}
p_{L}^{\nu}= & \frac{1}{2 p_{T}^{\ell 2}}\left\{\left[M_{W}^{2}+2\left(\vec{p}_{T}^{\ell} \cdot \vec{p}_{T}\right)\right] p_{L}^{\ell}\right. \\
& \left. \pm \sqrt{\left[M_{W}^{2}+2\left(\vec{p}_{T}^{\ell} \cdot \vec{p}_{T}\right)\right]^{2}\left|\vec{p}^{\ell}\right|^{2}-4\left(p_{T}^{\ell} E^{\ell} \dot{E}_{T}\right)^{2}}\right\}
\end{aligned}
$$

Consequently, there are two distinct estimates for the $W Z$ invariant mass which we label $M_{W Z}^{\mathrm{rec}, \max }$ and $M_{W Z}^{\mathrm{rec} \text { min }}$ the maximum and minimum reconstructed values, respectively. We show in left panel of Fig. 1 the $M_{W Z}^{\text {rec,max }}$ and $M_{W Z}^{\text {rec,min }}$, as well as the true $M_{W Z}$ invariant mass distributions for $M_{V_{1}^{ \pm}}=500 \mathrm{GeV}$. As seen in the figure, both

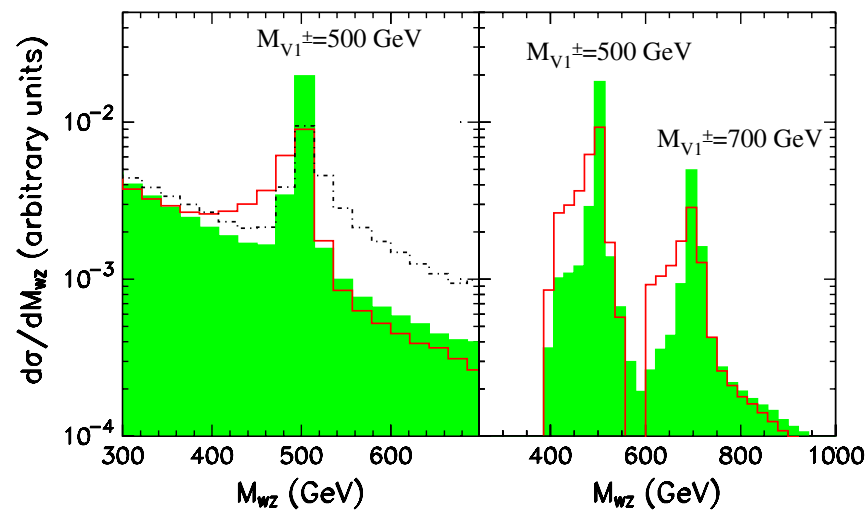

FIG. 1 (color online). Left: WZ invariant mass distribution reconstructed using the largest (dash-dotted line) and smallest (full line) estimate [see Eq. (9)], as well as the true distribution (shadowed region). Right: The true (shadow) and minimum (line) reconstructed $W Z$ invariant mass distributions after the cuts in Eq. (10), for two values of $M_{V_{1}^{ \pm}}$ reconstructed distributions present a clear peak associated to the presence of a new charged resonance. Moreover, the maximum (minimum) reconstructed $W Z$ invariant mass is a reasonable estimator of the true distribution for $W Z$ invariant masses smaller (larger) than the position of the resonance.

In order to isolate the contribution of the new charged states, we imposed a cut on $M_{W Z}^{\text {rec,min }}$

$400 \mathrm{GeV} \leq M_{W Z}^{\text {rec,min }} \leq 550 \mathrm{GeV}, \quad$ for $M_{V_{1}^{ \pm}}=500 \mathrm{GeV}$

$600 \mathrm{GeV} \leq M_{W Z}^{\text {rec,min }}, \quad$ for $M_{V_{1}^{ \pm}}=700 \mathrm{GeV}$.

The effect of these cuts on the $W Z$ invariant mass spectrum can be seen in the right panel of Fig. 1 for $M_{V_{1}^{ \pm}}=500 \mathrm{GeV}$ and $700 \mathrm{GeV}$. As seen in the figure after these cuts, a good fraction of the peak signal events are retained.

The predicted cross sections for the signal and SM backgrounds after cuts (5)-(10) are listed in Table I. From these numbers we conclude that the above cuts lead to a good signal to background ratio of $\simeq 2.4(1.5)$ for $M_{V_{1}^{ \pm}}=500(700) \mathrm{GeV}$. Thus, a clear observation $(5 \sigma)$ of the new charged resonances $V_{1}^{ \pm}$with a 500 (700) GeV mass in the leptonic channel requires a modest integrated luminosity of $15(66) \mathrm{fb}^{-1}$, which can be achieved in the low luminosity run of the LHC or in the early stages of the high luminosity run.

Similar sensitivity could be obtained by cutting, instead, on $M_{W Z}^{\text {rec,max }}$, though in general the cuts have to be chosen tighter and dependent on the $M_{V_{1}^{ \pm}}$mass. This is so because the SM background is a decreasing function of the $W Z$ mass, therefore when cutting on the maximum reconstructed $W Z$ mass, the number of miss-reconstructed background events in the signal region tends to be larger.

After the new state coupled to $W Z$ is discovered, it is important to probe its spin. The best way to accomplish that is to study angular correlations of the final state particles. In principle, useful information on the spin could be also extracted from the production cross section, however, at the LHC one measures the production cross section times the decay branching ratio, requiring additional information to disentangle these quantities. Here we employ two methods to unravel the spin of the new charged state based exclusively on the kinematic distribution of the final state particles. In the first method, we contrast the kinematic distributions of the charged leptons produced in the decay of vector and scalar charged states, much in the spirit of the analysis carried out to study the spin of supersymmetric particles at the LHC $[30,31]$. A virtue of this method is that it does not rely on the reconstruction of the neutrino momentum (besides the invariant mass cut). In our second analysis, we used the reconstructed neutrino momentum to obtain the polar angle of the produced $Z$ 's in the $W Z$ center-of-mass system.

In order to contrast the spin- 0 and spin- 1 resonances, we focused on the leptons whose momenta can be well deter- 
TABLE I. SM background and signal cross sections after different set of cuts in $\mathrm{fb}$. In the last column the top/bottom results is obtained applying the top/bottom cut in Eq. (10).

\begin{tabular}{llcccl}
\hline \hline & cuts (5)-(6) & cuts (5)-(7) & cuts (5)-(8) & cuts (5)-(10) \\
\hline $\mathrm{EW}$ & $W Z j j$ & 4.68 & 2.68 & 2.40 & $0.265 / 0.166$ \\
$t \bar{t} j$ & 22.4 & 6.54 & 1.85 & $0.024 / 0.0003$ \\
$M_{V_{1}^{ \pm}}=500 \mathrm{GeV}$ & 1.02 & 0.84 & 0.78 & 0.705 \\
$M_{V_{1}^{ \pm}}=700 \mathrm{GeV}$ & 0.36 & 0.32 & 0.30 & 0.25 \\
\hline \hline
\end{tabular}

mined. In previous studies [30], it has been shown that a convenient variable for such analysis is

$$
\cos \theta_{\ell \ell}^{*} \equiv \tanh \left(\frac{\Delta \eta_{\ell \ell}}{2}\right)
$$

where $\Delta \eta_{\ell \ell}$ is the rapidity difference between the same charge leptons. Notice that this quantity is invariant under longitudinal boosts. We plot in Fig. 2 the expected $\cos \theta_{\ell \ell}^{*}$ distributions for the SM background and the production of scalar and vector resonances with mass 500 (700) $\mathrm{GeV}$ in the left (right) panel after cuts (5)-(10). In obtaining this figure, we imposed that the cross section for the production of spin-0 resonances is the same of the one for spin-1 states. We also display the SM background alone to show its impact on the distributions.

These figures clearly show that the $\cos \theta_{\ell \ell}^{*}$ distribution for spin- 1 and spin-0 resonances are quite different and they can be used to quantify the required integrated luminosity needed to discriminate between them at a given CL. A simple $\chi^{2}$ analysis of the distributions shown in Fig. 2 yields a 99\% CL discrimination between spin-0 and spin-1 resonances of mass 500 (700) GeV for an integrated luminosity of $170(215) \mathrm{fb}^{-1}$, considering only the statistical errors.

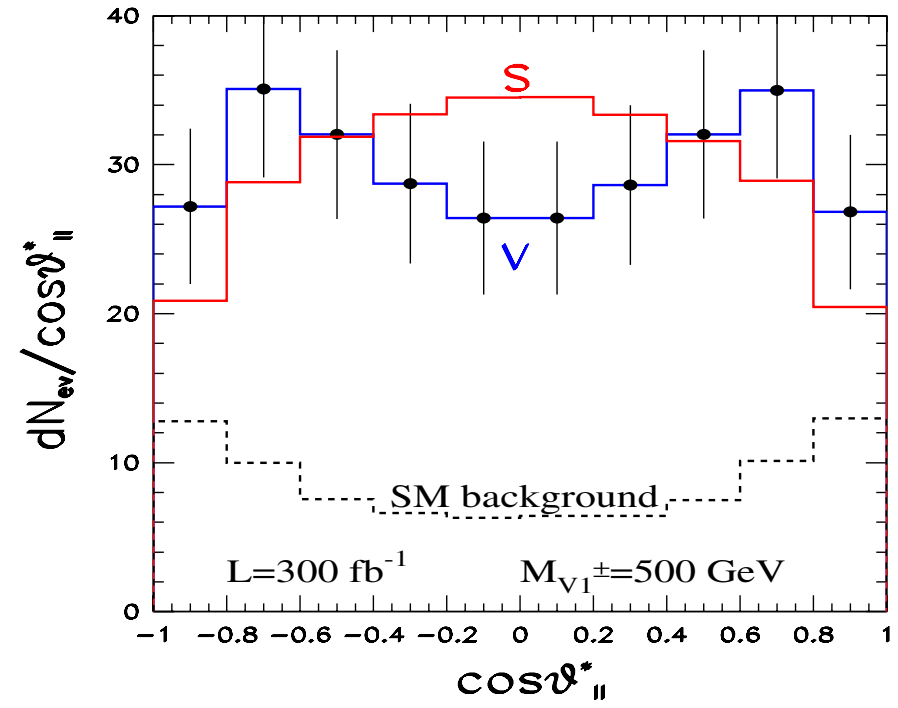

In order to eliminate possible normalization systematics in the angular distributions, we have also estimated the integrated luminosity needed to decipher the spin of the new charged state by constructing an angular asymmetry

$$
A_{\ell \ell}=\frac{\sigma\left(\left|\cos \theta_{\ell \ell}^{*}\right|<0.5\right)-\sigma\left(\left|\cos \theta_{\ell \ell}^{*}\right|>0.5\right)}{\sigma\left(\left|\cos \theta_{\ell \ell}^{*}\right|<0.5\right)+\sigma\left(\left|\cos \theta_{\ell \ell}^{*}\right|>0.5\right)}
$$

Considering only the statistical errors, this asymmetry allows a 99\% CL distinction between spin-0 and spin-1 resonances of mass 500 (700) GeV for an integrated luminosity of 440(560) $\mathrm{fb}^{-1}$. With these choices of integrated luminosities, we have $A_{\ell \ell}($ scalar $)=+0.104 \pm 0.05$ and $A_{\ell \ell}($ vector $)=-0.07 \pm 0.05$, for $M_{V_{1}^{ \pm}}=500 \mathrm{GeV}$, and $A_{\ell \ell}($ scalar $)=-0.036 \pm 0.06$ and $A_{\ell \ell}($ vector $)=-0.27 \pm$ 0.06 , for $M_{V_{1}^{ \pm}}=700 \mathrm{GeV}$, where we have quoted only the statistical errors.

We also studied the resolving power of the reconstructed $Z$ polar angle $\left(\theta_{W Z}\right)$ distribution evaluated in the $W Z$ center-of-mass frame. We display in Fig. 3 the $\cos \theta_{W Z}$ distribution for spin-1 charged states after cuts (5)-(10). Since the reconstructed neutrino momentum has a twofold ambiguity, there is also a twofold ambiguity in the reconstructed $Z$ polar angle in the $W Z$ center-of-mass frame

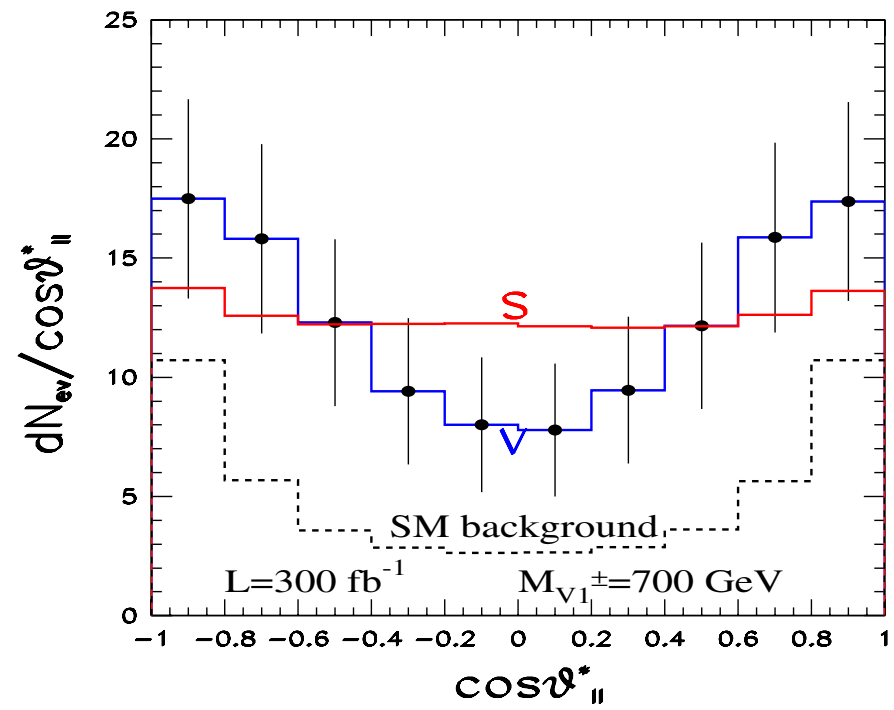

FIG. 2 (color online). $\quad \cos \theta_{\ell \ell}^{*}$ distribution for the SM background (dashed line), the production of a vector charged resonance (solid line with error bars), and the production of charged scalars (solid line). In the left (right) panel the mass of the new resonance is 500 (700) $\mathrm{GeV}$ and we considered an integrated luminosity of $300 \mathrm{fb}^{-1}$. 

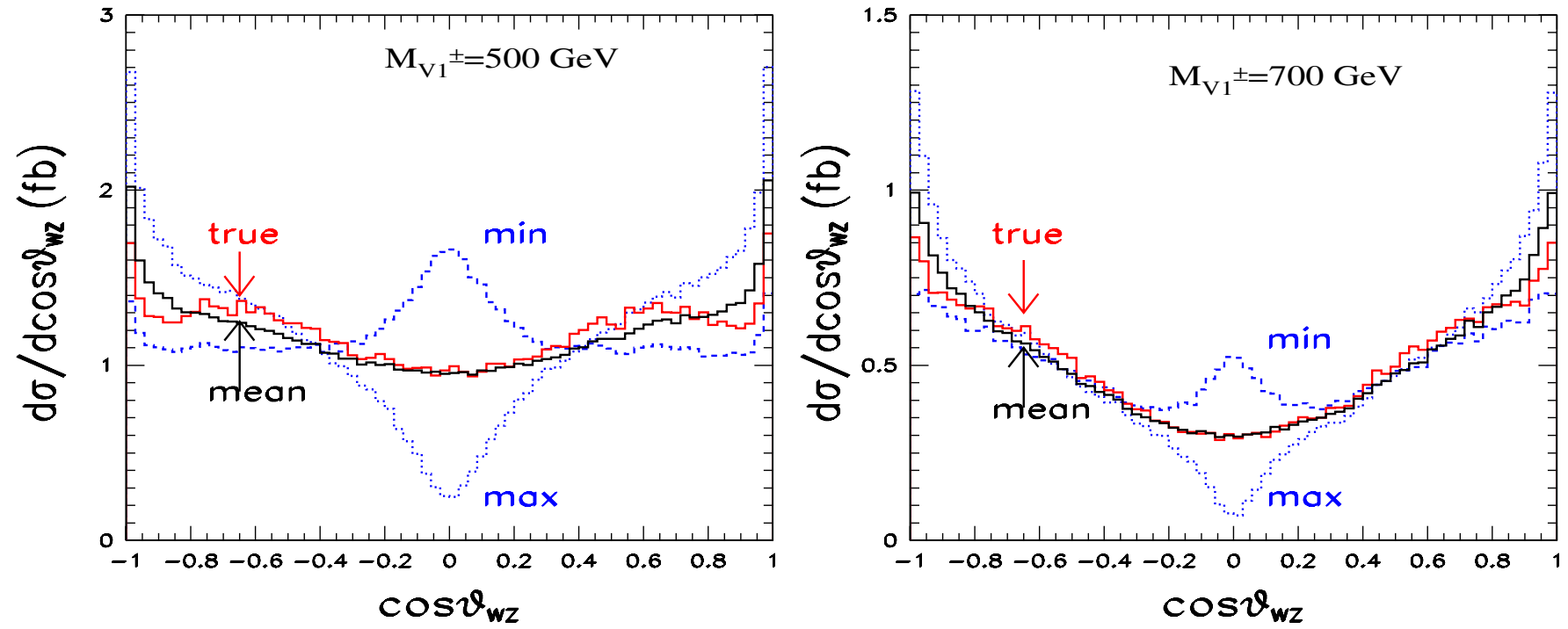

FIG. 3 (color online). $\quad \cos \theta_{W Z}$ distributions for a vector resonance of $500 \mathrm{GeV}$ (left panel) and $700 \mathrm{GeV}$ (right panel). The dashed (dotted) line stands for the reconstructed distribution using the neutrino momentum that leads to the maximum (minimum) $W Z$ invariant mass. The solid lines represent the true distribution (upper line) and the one obtained averaging the reconstructed distributions with maximum and minimum $W Z$ invariant mass (lower line).

which lead to the two distributions shown in the figure. The dashed (dotted) lines correspond to the reconstructed $Z$ polar angle distribution using the neutrino momentum that leads to the maximum (minimum) $W Z$ invariant mass. As we can see, the two distributions differ appreciably for $\cos \theta_{W Z}$ close to zero. However, as shown in the figure, the average of the two distributions has a better behavior in the central region of the detector and resembles the true distribution. Consequently, we have considered the average of the two reconstructed distributions as discriminating observable.

Figure 4 depicts such averaged distributions for charged vector and scalar resonances, where we are included the SM background prediction for assessment of its impact on

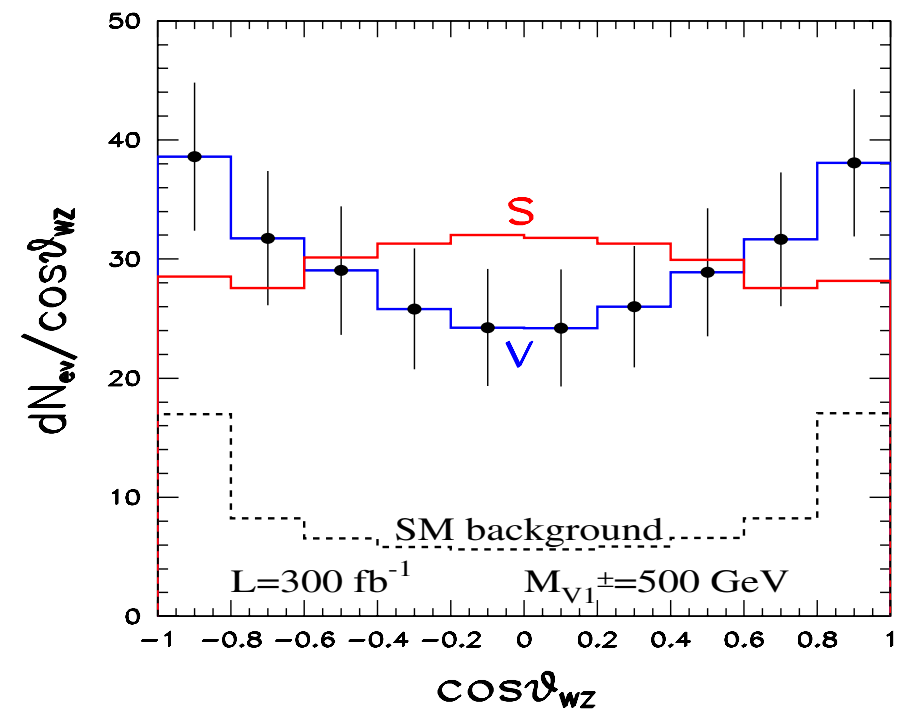

the spin determination. Clearly, the production of $V_{1}^{ \pm}$leads to more $W Z$ pairs produced at small polar angles while the scalar resonance leads to more central events, as expected. As above, in order to quantify the discriminating power between the scalar and vector productions we constructed the asymmetry

$$
A_{W Z}=\frac{\sigma\left(\left|\cos \theta_{W Z}\right|<0.5\right)-\sigma\left(\left|\cos \theta_{W Z}\right|>0.5\right)}{\sigma\left(\left|\cos \theta_{W Z}\right|<0.5\right)+\sigma\left(\left|\cos \theta_{W Z}\right|>0.5\right)}
$$

We find that for the new state mass of 500 (700) GeV, it is necessary $400(550) \mathrm{fb}^{-1}$ to separate the two possibilities at $99 \% \mathrm{CL}$. With these choices of integrated lumi-

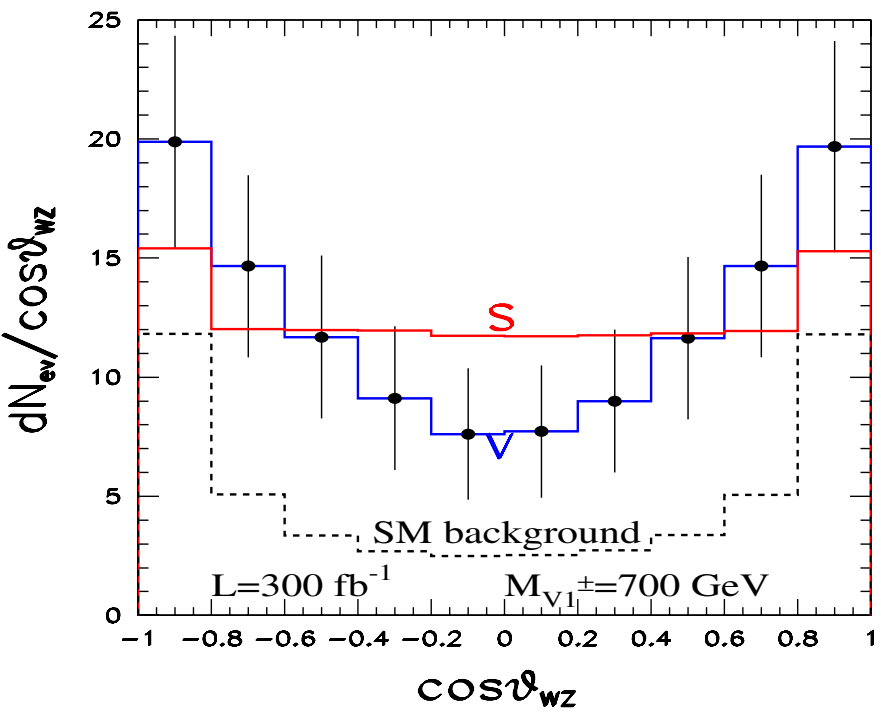

FIG. 4 (color online). Same as Fig. 2, but for the $\cos \theta_{W Z}$ distribution. 
nosities, we have $A_{W Z}($ scalar $)=+0.057 \pm 0.05$ and $A_{W Z}($ vector $)=-0.125 \pm 0.05$, for $M_{V_{1}^{ \pm}}=500 \mathrm{GeV}$, and $A_{W Z}($ scalar $)=-0.04 \pm 0.06 \quad$ and $\quad A_{W Z}$ (vector) $=$ $-0.28 \pm 0.06$, for $M_{V_{1}^{ \pm}}=700 \mathrm{GeV}$, where we have again quoted only the statistical errors. Furthermore the use of a $\chi^{2}$ analysis of the $\cos \theta_{W Z}$ distribution is able to reveal the spin of the new state at $99 \%$ CL for an integrated luminosity of $150(220) \mathrm{fb}^{-1}$, for $M_{V_{1}^{ \pm}}=500(700) \mathrm{GeV}$.

\section{CONCLUSIONS}

The observation of new charged vector resonances in Higgsless models decaying into $W Z$ pairs can be carried out via weak boson production at the LHC and their subsequent decays into charged leptons [28,29]. Here we show how the LHC will be able to determine the spin of these new states using two different methodologies. In the first method, only the observed charged leptons are used to discriminate between spin- 0 and spin- 1 resonances using the variable defined in Eq. (11). In this case, an integrated luminosity of $170(215) \mathrm{fb}^{-1}$ is needed to establish the spin of the 500 (700) $\mathrm{GeV}$ resonance at $99 \% \mathrm{CL}$ via a $\chi^{2}$ analysis of the $\cos \theta_{\ell \ell}^{*}$ distribution. On the other hand, the use of the asymmetry given by Eq. (12) requires $440(560) \mathrm{fb}^{-1}$ to determine the new resonance spin for a mass 500 (700) GeV. The second method is based on the two-folded reconstruction of the escaping neutrino momentum to obtain the $W Z$ polar angle distribution in its center-of-mass frame. This procedure requires a good understanding and calibration of the hadronic calorimeters, therefore, being subject to larger systematic uncertainties. We determined that the later method can distinguish between spin- 1 and spin- 0 states at $99 \%$ CL for integrated luminosities of $150(220) \mathrm{fb}^{-1}$ for $M_{V^{ \pm}}=500(700) \mathrm{GeV}$, respectively, when we perform a $\chi^{2}$ fit of the $\cos \theta_{W Z}$ distribution. If we use the asymmetry defined in Eq. (13) to perform the analysis, the integrated luminosities are 400 and $550 \mathrm{fb}^{-1}$, respectively. All results above only account for statistical errors and the inclusion of systematic uncertainties may render the efficiencies of the two methods rather similar.

\section{ACKNOWLEDGMENTS}

We thank G. Burdman for a careful reading of the manuscript. This work was supported in part by Conselho Nacional de Desenvolvimento Científico e Tecnológico (CNPq) and by Fundação de Amparo à Pesquisa do Estado de São Paulo (FAPESP); M. C. G.-G. is supported by National Science Foundation grant PHY0354776 and by Spanish Grants No. FPA-2007-66665C02-01, and No. FPA2006-28443-E.
[1] B. W. Lee, C. Quigg, and H. B. Thacker, Phys. Rev. Lett. 38, 883 (1977).

[2] B. W. Lee, C. Quigg, and H. B. Thacker, Phys. Rev. D 16, 1519 (1977).

[3] C. Csaki, C. Grojean, H. Murayama, L. Pilo, and J. Terning, Phys. Rev. D 69, 055006 (2004).

[4] C. Csaki, C. Grojean, L. Pilo, and J. Terning, Phys. Rev. Lett. 92, 101802 (2004).

[5] Y. Nomura, J. High Energy Phys. 11 (2003) 050.

[6] M.E. Peskin and T. Takeuchi, Phys. Rev. D 46, 381 (1992).

[7] G. Altarelli, R. Barbieri, and F. Caravaglios, Nucl. Phys. B405, 3 (1993).

[8] R. Barbieri, A. Pomarol, and R. Rattazzi, Phys. Lett. B 591, 141 (2004).

[9] G. Burdman and Y. Nomura, Phys. Rev. D 69, 115013 (2004).

[10] H. Davoudiasl, J.L. Hewett, B. Lillie, and T. G. Rizzo, Phys. Rev. D 70, 015006 (2004).

[11] G. Cacciapaglia, C. Csaki, C. Grojean, and J. Terning, Phys. Rev. D 70, 075014 (2004).

[12] H. Davoudiasl, J. L. Hewett, B. Lillie, and T. G. Rizzo, J. High Energy Phys. 05 (2004) 015.

[13] R. Barbieri, A. Pomarol, R. Rattazzi, and A. Strumia, Nucl. Phys. B703, 127 (2004).

[14] R. Foadi, S. Gopalakrishna, and C. Schmidt, J. High
Energy Phys. 03 (2004) 042.

[15] J. Hirn and J. Stern, Eur. Phys. J. C 34, 447 (2004).

[16] R. Casalbuoni, S. De Curtis, and D. Dominici, Phys. Rev. D 70, 055010 (2004).

[17] R.S. Chivukula, E. H. Simmons, H.-J. He, M. Kurachi, and M. Tanabashi, Phys. Rev. D 70, 075008 (2004).

[18] G. Cacciapaglia, C. Csaki, C. Grojean, and J. Terning, Phys. Rev. D 71, 035015 (2005).

[19] H. Georgi, Phys. Rev. D 71, 015016 (2005).

[20] R. Foadi, S. Gopalakrishna, and C. Schmidt, Phys. Lett. B 606, 157 (2005).

[21] R. Casalbuoni, S. De Curtis, D. Dolce, and D. Dominici, Phys. Rev. D 71, 075015 (2005).

[22] R. Sekhar Chivukula, E. H. Simmons, H.-J. He, M. Kurachi, and M. Tanabashi, Phys. Rev. D 72, 095013 (2005).

[23] R.S. Chivukula, E. H. Simmons, H.-J. He, M. Kurachi, and M. Tanabashi, Phys. Rev. D 72, 075012 (2005).

[24] G. Cacciapaglia, C. Csaki, G. Marandella, and J. Terning, Phys. Rev. D 75, 015003 (2007).

[25] R. Sekhar Chivukula, D. A. Dicus, and H.-J. He, Phys. Lett. B 525, 175 (2002).

[26] R.S. Chivukula, D. A. Dicus, H.-J. He, and S. Nandi, Phys. Lett. B 562, 109 (2003).

[27] R. Sekhar Chivukula, H. J. He, M. Kurachi, E. H. Simmons, and M. Tanabashi, Phys. Rev. D 78, 095003 
(2008).

[28] A. Birkedal, K. Matchev, and M. Perelstein, Phys. Rev. Lett. 94, 191803 (2005).

[29] H. J. He et al., Phys. Rev. D 78, 031701 (2008).

[30] A. J. Barr, Phys. Lett. B 596, 205 (2004); J. High Energy Phys. 02 (2006) 042.

[31] J. M. Smillie and B. R. Webber, J. High Energy Phys. 10 (2005) 069; A. Alves, O. Éboli, and T. Plehn, Phys. Rev. D 74, 095010 (2006); A. Alves and O. Éboli, Phys. Rev. D 75, 115013 (2007).

[32] K. Cheung, X.H. Wu, and Q.S. Yan, Phys. Rev. D 76,
116006 (2007).

[33] T. Stelzer and F. Long, Comput. Phys. Commun. 81, 357 (1994); F. Maltoni and T. Stelzer, J. High Energy Phys. 02 (2003) 027.

[34] J. Pumplin, D. R. Stump, J. Huston, H. L. Lai, P. Nadolsky, and W. K. Tung, J. High Energy Phys. 07 (2002) 012.

[35] O. J. P. Éboli, M. C. Gonzalez-Garcia, and J. K. Mizukoshi, Phys. Rev. D 74, 073005 (2006); A. S. Belyaev, O. J. P. Éboli, M. C. Gonzalez-Garcia, J. K. Mizukoshi, S. F. Novaes, and I. Zacharov, Phys. Rev. D 59, 015022 (1998). 